\title{
Comparison of 3D MR imaging sequences in knee articular cartilage at 1.5 T.
}

\author{
Mokhtar Mars*, Zeineb Tbini, Mouna Chelli, Fethi Ladeb
}

Université Tunis EL Manar, Institut Supérieur des Technologies Médicales de Tunis, Laboratoire de Recherche de Biophysique et de Technologies Médicales, 9, Avenue du Docteur Z. Essafi, Tunis, Tunisia

\begin{abstract}
Purpose: To compare 3D high-resolution MRI sequences in term of contrast, Signal to Noise Ratio (SNR), artefacts and cartilage thickness in knee articular cartilage at $1.5 \mathrm{~T}$.

Materials and methods: Fourteen healthy volunteers and seven patients underwent articular knee cartilage exam. The 3D sequences were VOLUME Imaging with Body Enhancement (VIBE), True Fast Imaging with Steady-state Precession (TRUEFISP), Dual Echo Steady State (DESS), Multi Echo Data Image Combination (MEDIC) and Sampling Perfection with Application optimized Contrasts using different flip angle Evolutions (SPACE). For all 3D sequences, we measured contrast ratio between cartilage-bone and cartilage-meniscus, SNR and cartilage thickness. In addition, we quantified magnetic susceptibility and flow artifacts. Statistical analyses were performed using the t-test and $\mathbf{P}<0.005$ was considered statistically significant.

Results: Statistical results were significantly different $(p>0.05)$. MEDIC and VIBE sequences showed the best contrast ratio between cartilage-meniscus $(0.40 \pm 0.14, p=0.000)$ and cartilage-bone $(0.75 \pm 0.4$, $\mathrm{p}=\mathbf{0 . 0 2 2})$, respectively. TRUEFISP sequence showed the highest SNR $(28.04 \pm 14.05, p=0.000)$ whereas DESS sequence showed the lowest SNR $(11.62 \pm 3.99, p=0.000)$. VIBE sequence provided the highest cartilage thickness measurement compared to the remaining 3D sequences $(2.0 \pm 0.4 \mathrm{~cm}$, IRR $=75.33 \%$, ICC=0.96). The quantification of magnetic susceptibility and flow artifacts revealed that TRUEFISP $(\mathrm{IRR}=\mathbf{5 9 \%}, \mathrm{ICC}=\mathbf{0 . 9 2})$ and SPACE $(\mathrm{IRR}=\mathbf{5 9 \%}, \mathrm{ICC}=\mathbf{0 . 9 6})$ sequences presented the highest artifacts compared to the others sequences.

Conclusion: 3D high-resolution sequences provide knee articular cartilage imaging with high image quality in relatively short acquisition time. MEDIC and VIBE sequences showed the best contrast and the lowest magnetic susceptibility and flow artifacts, TRUEFISP and SPACE sequence showed the highest SNR but they are more sensitive to artifacts, DESS sequence showed medium contrast and VIBE sequence provided the highest cartilage thickness.
\end{abstract}

Keywords: Knee articular cartilage, 3D high-resolution MRI sequences, Contrast, SNR, Artifacts.

Accepted on July 7, 2018

\section{Introduction}

Magnetic Resonance Imaging (MRI), with its excellent soft tissue contrast, is currently the imaging technique of choice for the assessment of articular cartilage [1]. To investigate Osteoarthritis (OA) of the knee joint with MRI, routine protocol includes a proton density weighted fat-suppression sequences in three planes (sagittal, coronal and axial) and a non-fat-suppressed T1-weighted sequence either in the sagittal or coronal plane.

2D MRI sequences such as Turbo Spin Echo (TSE) methods provide excellent signal-to-noise, good contrast between tissues in the knee articular cartilage and high in-plane spatial resolution. However, those sequences suffer from some limitations. First, because of using a slice thickness of about 3 $\mathrm{mm}$, they suffer from partial-volume artifacts. Second, the difficulty to evaluate oblique and small structures because cartilage is a thin layer with a complex surrounding anatomical structure such as ligaments and tendons which are oriented oblique and difficult to assess with 2D sequences.

3D high-resolution imaging is suggested to be an alternative of $2 \mathrm{D}$ imaging since it provides isotropic or nearly isotropic 3D imaging which can be reconstructed in any desired orientation with contiguous thin slices in order to reduce partial volume artifact effects [2-4]. The use of Multi-channel extremity coils at higher field strengths provides higher signal and contrast to Noise ratio, which allow acquiring isotropic images at short scanning time [5]. In addition, using auto-Allign technique, this type of sequence allows good reproducibility especially in case of follow-up treatment after an injury or cartilage repair or when a reproducible location is required.

The differences between 3D sequences are mostly related to the timing of the gradient pulses, the radio frequency excitation pulses and the measurement of echoes which leads to a 
different contrast between tissues. In order to use 3D sequence as routine sequence in the place of the three $2 \mathrm{D}$ sequences in different planes, the acquisition time of the 3D sequences should not exceed too much the timing of all three 2D sequences together.

There are many types of 3D high resolution sequences including 3D Gradient Echo with destruction of the residual transverse magnetization (VIBE), balanced steady-state free precession sequence (TRUEFISP), Dual Echo steady state gradient echo sequence (DESS), gradient echo sequence where multiple echoes are collected from a single excitation (MEDIC) and 3D spin echo Sampling Perfection with Application optimized Contrasts using different flip angle Evolutions (SPACE) [6-9].

The VIBE sequences are gradient echo sequences with destruction of the transverse signal by adding spoiler gradient along the slice and readout direction. The high speed data acquisition using data interpolation reduces respiratory and movement artifacts [5,10]. The TRUEFISP sequence is a balanced steady-state free precession sequence. With this technique, fluid has high signal, while cartilage signal intensity is preserved, resulting in excellent contrast.

In DESS sequence, we acquire two gradient echoes which are combined to provide a higher $\mathrm{T}_{2}{ }^{*}$ weighting with good contrast between cartilage and synovial fluid [11]. The DESS sequence is characterized by a short Repetition Time (TR) which will limit the decay of transverse magnetization before applying the next RF pulse [12].

The MEDIC sequence is a gradient echo sequence where multiple echoes are collected from a single excitation. The fact to collect and to combine several echoes of the same line of encoding allows being less sensitive to motion artifacts while increasing the SNR of the image. This sequence is less sensitive to movement and flow artifacts. The SPACE sequence is a special $3 \mathrm{D}$ TSE sequence with $\mathrm{T}_{1} \mathrm{w}$ contrast that allows an improved anatomical diagnostic in addition to the verification of findings in the $\mathrm{T}_{2} \mathrm{~W}$ sequence [13].

The aim of this study was to compare 3D sequences in the assessment of knee articular cartilage at $1.5 \mathrm{~T}$ in terms of contrast between cartilage and meniscus or bone and signal to noise. In addition, we will compare cartilage thickness measurement and quantify the magnetic susceptibility and flow artifacts between all 3D sequences.

\section{Material and Methods}

This study was approved by the ethics commission of our institute, verbal and written consent was obtained from all patients prior to exam.

\section{Population}

Twenty-one subjects were scanned between August 2017 and January 2018. Fourteen healthy volunteers without clinically evident of knee pathology ( 7 males and 7 females, mean age $33.19 \pm 11.04 \mathrm{y})$. Exclusion criteria's include a history of cartilage tears or major trauma of the knee. The asymptomatic volunteers were randomized for study of either the right or left Knee. Seven symptomatic patients with knee diseases ( 7 males mean age $30.42 \pm 12.39$ y) were prospectively examined. Inclusion criteria's were chronic or traumatic or degenerative knee pathology. The exclusion criteria for all subjects were contraindications to MR imaging.

\section{MR exam}

MR examination was performed using 1.5 T MR Scanner (Magnetom Area, Siemens Erlangen, Germany) equipped with 15 channels knee coil. All subjects were positioned in the isocenter of the magnet, feet-first, supine position and both knee in flexion position. The knee joint was well positioned in the center of the coil and was tightly fixed with fitting cushions to avoid motion. MR imaging protocol of the knee included a sagittal $\mathrm{T}_{1}$-weighted Turbo Spin Echo (TSE T1) as well as sagittal, axial and coronal PD-weighted fat suppression Turbo Spin Echo (TSE PD FS) and the 3D high resolution sequences (VIBE, TRUEFISP, DESS, MEDIC, SPACE). To compare the $3 \mathrm{D}$ high resolution sequences in an efficient way, we used the same geometrical parameters and almost the same bandwidth (same chemical shift) when it's possible. For the geometrical parameters, we used a square Field of View (FOV) of $160 \mathrm{~mm}$ $\times 160 \mathrm{~mm}$, a slice thickness of $0.6 \mathrm{~mm}$, a number of slices equal to 96 to cover almost the whole knee, contiguous slices and the acquisition matrix was $256 \times 256$. The phase encoding direction was Anterior-Posterior. The orientation was the sagittal plane. The bandwidth was around $220 \mathrm{~Hz} /$ pixel, which correspond to a chemical shift of 1 pixel at $1.5 \mathrm{~T}$. To reduce the scan time, we used the Grappa Parallel Acquisition Technique with an acceleration factor of 2 . The details of the specific scanning parameters for each 3D high-resolution sequence are listed in Table 1.

\section{Images analysis}

Morphologic MRI evaluation was realized for all subjects on TSE T1 sagittal images as well as on sagittal, axial and coronal TSE PD FS images by a radiologist expert in musculoskeletal MRI (23 years of experience) to identify the knee pathology and confirm the inclusion and exclusion criteria's in all subjects.

Images analysis of knee articular cartilage was performed on sagittal plane using Siemens workstation with syngo software by a radiologist (Figure 1). First, three regions of interest (ROI's) were manually drawn in the anterior, median and posterior femoral articular cartilage on one image of one sequence, and then copied to other sequences. Figure 2 showed the representation of evaluated three regions of interest on knee articular cartilage.

We calculated the Contrast ratio (C) between the different 3D high resolution sequences, based on the absolute value of signals difference between cartilage in three ROI's (Anterior, median and posterior) and bone or meniscus divided by the sum of both signals intensities [14]. 


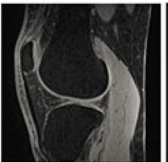

VIBE

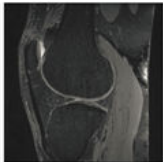

TRUEFISP

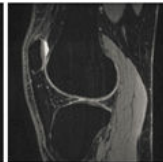

DESS

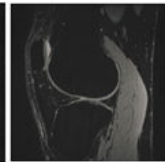

MEDIC

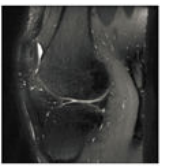

SPACE
Figure 1. 3D high resolution images of knee articular cartilage of 26 $y$ old healthy volunteer.

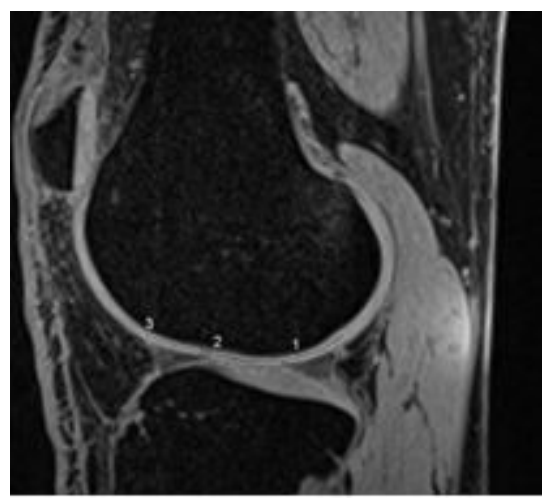

Figure 2. Representation of the evaluated three regions of interest on knee articular cartilage: (1) the posterior ROI; (2) the median ROI; (3) the anterior ROI.

To compare SNR between sequences, ROI's were placed in the different cartilage regions to measure the signal intensity and in the background of the image to measure the Standard Deviation (SD). The SNR in each region was calculated by the ratio of the mean signal intensity of cartilage divided by the SD of Background. The ROI's were drawn in the cartilage regions (Anterior, median and posterior). Then, we compared the SNR value in each region between all $3 \mathrm{D}$ high resolution sequences.

To measure the cartilage thickness, we have drawn three perpendicular tangents to the cartilage bone interface in the anterior, median and posterior cartilage region. Cartilage thickness was calculated using the distance tool in the three regions. The cartilage thickness measurements between all sequences were done by displaying the sequences side by side in the monitor. For identical placement, ROI's were copied from the first sequence and pasted on the remaining ones. Then, the distance is adjusted to match the anatomy displayed by the sequence.

For the quantification of 3D sequences in terms of artifacts, we displayed all sequences images in the same layout on the system monitor. Then we have chosen the most altered image among all sequences (same slice position for all sequences). The qualitative artifacts measurement in the different 3D sequences was scaled using five-point scale going from 5 to 1 with 5 belongs to the highest artifacts sequence and 1 is the lowest artifact sequence.

\section{Inter and Intra rater's repeatability}

To calculate Inter and Intra rater's repeatability variation for cartilage thickness and artifacts measurement, all twenty-one subjects were evaluated by two independent experienced raters
(3 years and 2 years of experience respectively). Intra rater repeatability was calculated from two independent evaluations of fifteen subjects by one observer ( 2 years of experience), with a delay of $1 \mathrm{w}$ between the two evaluations, and it was expressed as an Intra Class Correlation coefficient (ICC). In our study, ICC $>0.75$ means a very good reproducibility and $0.40<$ ICC $>0.75$ means reproducibility medium to good.

To calculate the Inter-Rater Reliability coefficient in percent (IRR (\%)), we based on the calculation of the percent of agreement among raters. First, we calculate the number of ratings that are in agreement. Second, we calculate the total number of ratings. Then, the percent agreement equal to the number of agreement ratings divided by the total number of ratings times 100. Minimal agreement was set less than $70 \%$ and high agreement was set more than $90 \%$.

\section{Statistical analysis}

All measurements of contrast, SNR and cartilage thickness are expressed in mean values \pm standard deviation. Inter and intraobserver reproducibility was evaluated by ICC method and IRR. Statistical analysis was performed with SPSS version 15 software. Statistical analyses were performed using the t-test and $\mathrm{P}<0.005$ was considered statistically significant.

\section{Results}

The results of cartilage-bone contrast evaluation have shown that VIBE and SPACE sequences demonstrated the best contrast, followed by the DESS and MEDIC sequences which presented a medium contrast and the TRUEFISP sequence which showed the lowest contrast. For cartilage-meniscus contrast evaluation, the MEDIC and SPACE sequences presented the best contrast whereas the DESS and VIBE sequences showed the medium contrast and the lowest contrast was obtained using the TRUEFISP sequence. The results of mean contrast between cartilage and bone or meniscus for 3 Dhigh resolution sequences in different regions are summarized in Table 2. Figure 3 shows diagram of mean contrast ratio between cartilage and bone or meniscus for $3 \mathrm{D}$ sequences.

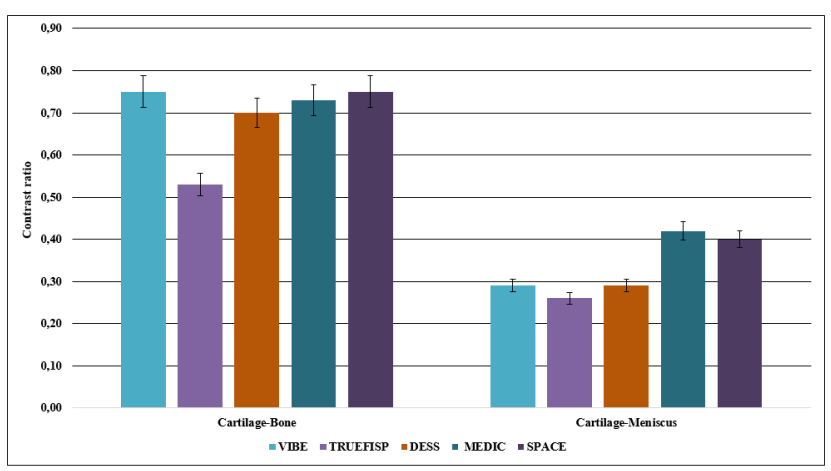

Figure 3. Diagram of mean contrast ratio between cartilage and lesion, bone and meniscus of $3 D$ high resolution sequences.

For SNR analysis, VIBE and TRUEFISP sequences showed clearly higher SNR compared to the other 3D sequences. DESS 
sequence showed the lowest SNR and the medium SNR was present by the MEDIC and SPACE sequences. The details of mean SNR cartilage for 3D sequences are summarized in Table 3. Diagram of mean SNR for $3 \mathrm{D}$ sequences is displayed in Figure 4.

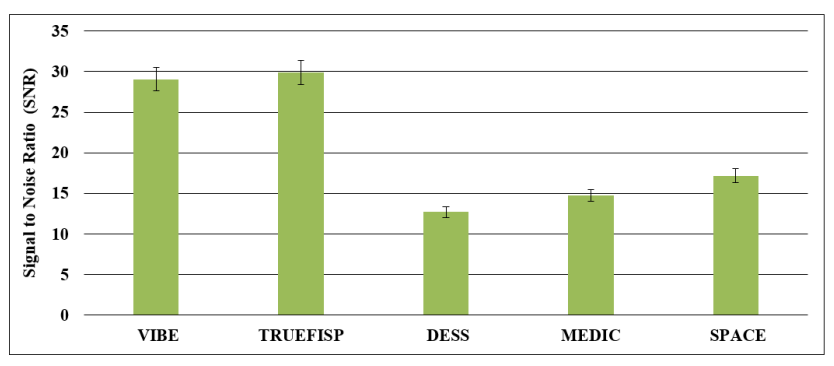

Figure 4. Diagram of mean SNR of $3 D$ high resolution sequences.

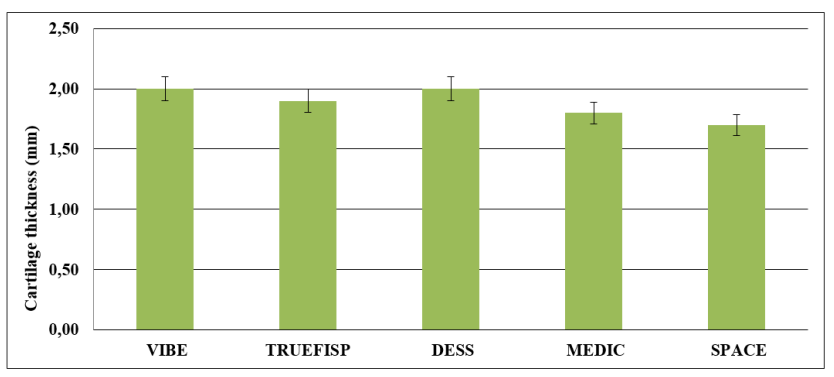

Figure 5. Diagram of mean cartilage thickness of $3 D$ high resolution sequences.

Table 1. Scanning parameters for 3D high resolution sequences used for morphological assessment of the articular cartilage.

\begin{tabular}{llllll}
\hline Parameters & VIBE & TRUEFISP & DESS & MEDIC & SPACE \\
\hline Repetition time (ms) & 14.5 & 11 & 20.12 & 36 & 1000 \\
\hline Echo time (ms) & 6.07 & 4.88 & 7.24 & 20 & 24 \\
\hline Flip angle $\left(^{\circ}\right)$ & 10 & 28 & 25 & 8 & 28 \\
\hline Bandwidth (Hz/pixel) & 180 & 181 & 181 & 181 & 181 \\
\hline Slice oversampling (\%) & 25 & 100 & 25 & 25 & 100 \\
\hline Acquisition time (min: s) & $02: 07$ & $02: 32$ & $02: 55$ & $05: 12$ & $05: 43$ \\
\hline
\end{tabular}

Cartilage thickness measurement revealed that VIBE sequence has higher cartilage thickness compared to the other $3 \mathrm{D}$ sequences with a good agreement inter and intra raters reproducibility (IRR $=75.33 \%$ and $\mathrm{ICC}=0.96)$. The intraobserver reproducibility measurements of cartilage thickness were good for all 3D sequences $(\geq 0.75)$. And, the inter-raters reproducibility measurements showed lower agreement between the two raters $(\leq 70 \%)$ for all sequences. There was a good agreement between the two raters for all $3 \mathrm{D}$ sequences. Mean, SD, IRR and ICC cartilage thickness of 3D high resolution sequences are summarized in Table 4. Figure 5 shows the diagram of the mean cartilage thickness for $3 \mathrm{D}$ sequences.

The quantification of magnetic susceptibility artifact in $3 \mathrm{D}$ sequences revealed that TRUEFISP sequence presented the highest artifact followed by the SPACE sequence. For the remaining 3D sequences there were minimal artifacts. The inter-raters reproducibility showed a minimal agreement between the two raters $(\leq 70 \%)$ for all sequences. There was a high agreement between the two raters for VIBE, DESS and MEDIC sequences compared to TRUEFISP and SPACE sequences. The intra-observer reproducibility of evaluations was good for all 3Dhigh resolution sequences $(\geq 0.75)$.

Table 2. Summary of mean contrast between cartilage and bone or meniscus in different cartilage regions.

\begin{tabular}{|c|c|c|c|c|c|c|c|c|}
\hline \multirow{2}{*}{$\begin{array}{l}\text { 3D high } \\
\text { resolution } \\
\text { Sequences }\end{array}$} & \multicolumn{4}{|c|}{ Bone } & \multicolumn{4}{|c|}{ Meniscus } \\
\hline & Mean & A & M & $\mathbf{P}$ & Mean & A & $\mathbf{M}$ & $\mathbf{P}$ \\
\hline VIBE & 0.75 & 0.75 & 0.75 & 0.75 & 0.27 & 0.27 & 0.27 & 0.26 \\
\hline TRUEFISP & 0.54 & 0.54 & 0.54 & 0.54 & 0.25 & 0.25 & 0.28 & 0.25 \\
\hline DESS & 0.71 & 0.71 & 0.71 & 0.70 & 0.27 & 0.28 & 0.27 & 0.26 \\
\hline MEDIC & 0.72 & 0.72 & 0.72 & 0.72 & 0.41 & 0.41 & 0.41 & 0.40 \\
\hline SPACE & 0.67 & 0.67 & 0.68 & 0.68 & 0.36 & 0.37 & 0.36 & 0.35 \\
\hline
\end{tabular}

Table 3. Summary of mean SNR in different cartilage regions.

\begin{tabular}{lllll}
\hline 3D high resolution sequences & Mean (SD) & $\mathbf{A}(\mathrm{SD})$ & $\mathbf{M}(\mathrm{SD})$ & $\mathbf{P}(\mathrm{SD})$ \\
\hline VIBE & 25.45 & 25.70 & 25.40 & 25.11 \\
\hline TRUEFISP & 27.93 & 28.18 & 27.50 & 26.56 \\
\hline DESS & 11.46 & 11.82 & 11.55 & 11.23 \\
\hline MEDIC & 12.34 & 12.55 & 12.01 & 12.01 \\
\hline SPACE & 15.62 & 15.93 & 15.04 & 15.04 \\
\hline
\end{tabular}

The quantification of flow artifact in all 3D sequences demonstrated that TRUEFISP sequence presented the highest artifact followed by the SPACE sequence. For the other 3D sequences, there were minimal artifacts. The inter-raters reproducibility showed a minimal agreement between the two raters $(\leq 70 \%)$ for all sequences. There was a high agreement between the two raters for VIBE, DESS and MEDIC sequences compared to TRUEFISP and SPACE sequences. The intraobserver reproducibility of readings was good for all $3 \mathrm{D}$ sequences $(\geq 0.75)$. ICC and IRR measurements of $3 \mathrm{D}$ sequences flow and magnetic susceptibility artifacts are summarized in Table 5.

Table 4. Summary of mean slice thickness, ICC and IRR in different cartilage regions of $3 D$ high resolution sequences.

\begin{tabular}{lllll}
\hline 3D high resolution sequences & Measured parameters & Mean & A & M
\end{tabular}




\begin{tabular}{|c|c|c|c|c|c|}
\hline \multirow[t]{3}{*}{ VIBE } & Mean (SD) & $2.0(0.4)$ & $2.2(0.4)$ & $1.8(0.5)$ & $2.2(0.4)$ \\
\hline & IRR & $75.33 \%$ & $73 \%$ & $80 \%$ & $73 \%$ \\
\hline & ICC & 0.96 & 0.98 & 0.96 & 0.96 \\
\hline \multirow[t]{3}{*}{ TRUEFISP } & M (SD) & $1.9(0.4)$ & $2.0(0.4)$ & $1.7(0.5)$ & $2.1(0.3)$ \\
\hline & IRR & $57.33 \%$ & $66 \%$ & $46 \%$ & $60 \%$ \\
\hline & ICC & 0.93 & 0.96 & 0.97 & 0.88 \\
\hline \multirow[t]{3}{*}{ DESS } & $M(S D)$ & $2(0.3)$ & $2.0(0.3)$ & $1.7(0.4)$ & $2.3(0.3)$ \\
\hline & IRR & $68.33 \%$ & $66 \%$ & $66 \%$ & $73 \%$ \\
\hline & ICC & 0.91 & 0.92 & 0.93 & 0.88 \\
\hline \multirow[t]{3}{*}{ MEDIC } & $M(S D)$ & $1.8(0.4)$ & $1.9(0.4)$ & $1.6(0.4)$ & $2.1(0.4)$ \\
\hline & IRR & $71 \%$ & $73 \%$ & $60 \%$ & $80 \%$ \\
\hline & ICC & 0.97 & 0.97 & 0.97 & 0.98 \\
\hline \multirow[t]{3}{*}{ SPACE } & $M(S D)$ & $1.7(0.3)$ & $1.6(0.4)$ & $1.5(0.3)$ & $2.0(0.4)$ \\
\hline & IRR & $49 \%$ & $60 \%$ & $33 \%$ & $54 \%$ \\
\hline & ICC & 0.98 & 0.98 & 0.98 & 0.99 \\
\hline
\end{tabular}

Mean: mean value of the 3 ROI's, SD: Standard Deviation; A: Anterior, M: Median; P: Posterior; IRR: Inter Rater repeatability; ICC: Intra Class Correlation coefficient.

Table 5. Summary of IRR and ICC measurements of magnetic susceptibility and flow artifacts between $3 D$ high resolution sequences.

\begin{tabular}{llllll}
\hline \multirow{2}{*}{ 3D high resolution sequences } & \multicolumn{3}{l}{ Magnetic susceptibility } & Flow \\
\cline { 2 - 5 } & ICC & IRR & ICC & IRR \\
\hline VIBE & 0.90 & $63 \%$ & 0.96 & $63 \%$ \\
\hline TRUEFISP & 0.95 & $45 \%$ & 0.92 & $59 \%$ \\
\hline DESS & 0.79 & $59 \%$ & 0.97 & $63 \%$ \\
\hline MEDIC & 0.97 & $720 \%$ & 0.93 & $68 \%$ \\
\hline SPACE & 0.92 & $59 \%$ & 0.96 & $59 \%$ \\
\hline
\end{tabular}

IRR: Inter Rater repeatability; ICC: Intra Class Correlation coefficient.

\section{Discussion}

3D sequences have the potential of isotropic imaging. They provide high image quality (SNR, contrast, spatial resolution) in a relatively short acquisition time. Recently, several studies have investigated 3D sequences performance for assessment of articular cartilage knee disease [7-9,15,16].

When comparing contrast between cartilage and surrounding tissues between 3D sequences we noticed in our study that VIBE and SPACE sequences have the best cartilage-bone contrast and the MEDIC sequence has the best cartilagemeniscus contrast whereas The TRUEFISP sequence has the lowest cartilage bone and cartilage meniscus contrast. 3D DESS sequence has an intermediate contrast between cartilage and bone or meniscus.
A previous study reported that the highest SNR and cartilagesynovial fluid CNR were obtained with a flip angle of $60^{\circ}$ when using DESS sequence [17]. Zhuo-Zhao found that fatsuppressed 3D VIBE can achieve images with higher cartilage SNR, higher CNR between the cartilage and the surrounding tissues, and reduced pulsation artifact in a much shorter acquisition time [6]. Osameh and Louise reported that TRUEFISP at $3 \mathrm{~T}$ provided excellent cartilage-joint fluid contrast and that cartilage is better visualized with TRUEFISP than SPACE sequences $[9,15]$. Another study demonstrated that the disadvantage of SPACE was the poor cartilage-to-fluid contrast and less ability for distinctions between cartilage and other surrounding tissues [18]. In a previous study, it was reported that traditional three-dimensional gradient echo (GRE) has less contrast between cartilage and synovial fluid compared with spin echo technique $[19,20]$. Lee et al. have reported that fat suppressed 3D gradient echo sequences are better than $2 \mathrm{D}$ fat suppressed proton density sequences for differentiating grade 3 and grade 4 articular cartilage defects [21].

A previous study reported that the contrast between joint fluid and cartilage (measured as CNR) was highest for 3D WE true FISP sequences when compared with two-dimensional (2D) intermediate-weighted spin-echo with fat saturation, 2D fast Short Inversion Time Inversion-Recovery (STIR), 3D WE DESS, and 3D fat saturated Fast Low Angle Shot (FLASH) sequences [22]. A study reported that TrueFISP allows assessment of the knee articular cartilage abnormalities with moderate-to-high specificity and low-to-moderate sensitivity [23]. The SPACE was reported to have the same abnormality detection when compared with conventional 2D TSE and 
intersequence- and interreader correlation showed no significant differences [24].

The contrast in $3 \mathrm{D}$ sequences depends mostly on the TR, TE, flip angle, the refocusing process and timing of the echo measurement. So, variation in different results of previous study is mainly explained by the difference in sequence parameters and magnetic field strength.

The SNR depends on many parameters such as sequence type, sequence parameters, Hardware (coil architecture) and magnetic field strength. Since we were using the same equipment at the same magnetic field, the difference in SNR will be related mostly to sequence type and sequence parameters. In our study, TRUEFISP and VIBE sequences have shown the highest SNR followed by 3D SPACE, 3D MEDIC and 3D DESS sequences. The SNR for gradient echo sequence depends mostly on the TR, TE, the flip angle, voxel size, the bandwidth, the refocusing process and the magnetic field strength. In our study, those combinations made from the VIBE and TRUEFISP sequences the sequences with highest SNR. A previous study reported that 3D SPACE has better SNR compared with other 3D morphology sequences commonly used for cartilage assessment $[4,8,9,25]$. The SNR and contrast of the 3D SPACE could be improved by the Radial k-space acquisition and elliptical scanning $[15,18]$. In medical imaging, it is often desirable to perform some kind of noise reduction to improve Image behaviour and make diagnostic much easier. The nonlinear digital filtering technique using median filter is often employed for this purpose [26].

To detect easily the cartilage boundary, we need relatively high in-plane resolution and high contrast between the cartilage and adjacent subchondral bone. The articular cartilage thickness is only $1.3-2.5 \mathrm{~mm}$ in healthy knees and even less in knees with Osteoarthritis (OA); it's required to have high spatial resolution to ensure a sufficient number of pixels for better measurement accuracy of cartilage thickness [27,28].

In terms of cartilage thickness evaluation, VIBE sequence showed higher cartilage thickness measurement compared to the other 3D high resolution sequences with a good agreement inter and intra raters reproducibility (IRR $=75.33 \%$ and $\mathrm{ICC}=0.96$ ). DESS was reported to have similar sensitivity to changes in knee cartilage thickness over time when compared with other 3D GRE techniques tested in longitudinal knee osteoarthritis trial [29]. The same sequence has been reported to be reliable in the assessment of cartilage thickness and volume with good accuracy and precision [30].

To compare the accuracy of slice thickness, we need to use another standard evaluation tool of cartilage thickness and to compare the results with those obtained by all 3D sequences.

The acquisition time in MRI depends on many parameters such as TR, matrix in the phase encoding direction, the acceleration factor in case of Fast Spin Echo sequence, number of excitation and number of slices in case of $3 \mathrm{D}$ sequences which is the case. We tried to keep the same geometrical parameters (matrix). The difference between timing of the sequences is mostly because of TR which the parameter that consumes more the time and the oversampling in the slice direction which is used to remove aliasing artifact in the slice encoding direction (the slice profile in not rectangular).

The spin echo sequence family has the longest acquisition time because of the use of long TR. The SPACE sequence belongs to this family. The MEDIC sequence which is a gradient echo sequence, known with its shorter TR compared to the spin echo sequence has a long acquisition time because of the oversampling in the slice direction to avoid aliasing artifact. To keep the acquisition time in acceptable range, we used the parallel acquisition technique with an acceleration factor equal to two. A previous study done on articular cartilage of the ankle reported that the TRUEFISP sequence with high isotropic resolution of $0.3 \mathrm{~mm}$ provided the shortest scan time compared to all 3D GRE sequences [31].

Our study has some limitations. First, the numbers of subjects are small and the population is heterogeneous. Second, several factors including the magnetic field strength, the methods of parameters calculation and the unavoidable experimental noises can explain the differences of our findings compared to other previously published results. Another limitation of our study could be the partial volume effect and chemical shift artifact. The reduction of the partial volume effect and the chemical shift artifact which affect the cartilage-bone interface can be reduced in theory using thin slices and with fat suppression technique using water excitation or spectral fat suppressed methods which allow an accurate measurement of the articular cartilage thickness [32]. In our study, we used the water excitation fat saturation technique and a bandwidth of about $220 \mathrm{~Hz}$ to reduce the chemical shift artifact. Last limitation of our study is that we compared the slice thickness only between $3 \mathrm{D}$ sequences and not using a standard slice thickness tools.

It is important to note that image quality parameters such as SNR, contrast and criteria's like spatial resolution and acquisition time are linked together, so it's difficult to improve one parameter without changing other parameters. Then, it's not easy to find a balance among all those parameters to obtain optimal delineation of a given joint like the articular knee cartilage in our case. Future researches are recommended to evaluate the performance of optimized 3D sequences at higher magnetic field to find the best sequence in the early diagnosis of osteoarthritis with minimal artifacts.

\section{Conclusion}

3D high resolution sequences provide knee articular cartilage imaging with high image quality (SNR, contrast, artifacts) in relatively short acquisition time, minimum artifacts and more accurate cartilage thickness measurement. MEDIC and VIBE sequences showed the best contrast, TRUEFISP and SPACE sequence showed the highest SNR but they are more sensitive to artifacts compared to the others 3D sequences, DESS sequence showed the lowest SNR, VIBE sequence provided 
the highest cartilage thickness measurement compared to the remaining $3 \mathrm{D}$ sequences

\section{Conflict of Interest}

The authors declare that they have no conflict of interest.

\section{Acknowledgements}

None

\section{References}

1. Gold GE, Chen CA, Koo S, Hargreaves BA, Bangerter NK. Recent advances in MRI of articular cartilage. AJR Am J Roentgenol 2009; 193: 628-638.

2. Duc SR. Improved visualization of collateral ligaments of the ankle: multiplanar reconstructions based on standard 2D turbo spin-echo MR images. Eur Radiol 2007; 17: 1162-1171.

3. Gold GE, Busse RF, Beehler C, Han E, Brau AC, Beatty $\mathrm{PJ}$, Beaulieu CF. Isotropic MRI of the knee with 3D fast spin-echo extended echo-train acquisition (XETA): initial experience. AJR Am J Roentgenol 2007; 188: 1287-1293.

4. Kijowski R, Blankenbaker DG, Klaers JL, Shinki K, De Smet AA, Block WF. Vastly undersampled isotropic projection steady-state free precession imaging of the knee: diagnostic performance compared with conventional MR. Radiology 2009; 251: 185-194.

5. Kornaat PR, Reeder SB, Koo S, Brittain JH, Yu H, Andriacchi TP, Gold GE. MR imaging of articular cartilage at 1.5T and 3.0T: comparison of SPGR and SSFP sequences. Osteoarthritis Cartilage 2005; 13: 338-344.

6. Zhuo ZZ, Han S, Xuan L. Fat-suppressed 3D T1-weighted gradient-echo imaging of the cartilage with a volumetric interpolated breath-hold examination. Am J Roentgenol 2010; 194: 414-419.

7. Altahawi FF, Blount KJ, Morley NP, Raithel E, Omar IM. Comparing an accelerated 3D fast spin- echo sequence (CS-SPACE) for knee 3T magnetic resonance imaging with traditional 3D fast spin-echo (SPACE) and routine 2D sequences. Skelet Radiol 2010; 46: 7-15.

8. Boris B, Wolfgang L, Julian LW, Sebastian F, Thomas JV, Martin B. Chondral lesions in the patellofemoral joint in MRI: Intra-individual comparison of short-tau inversion recovery sequence (STIR) with $2 \mathrm{D}$ multiple-echo data image combination sequence (MEDIC). Eur J Radiol 2016; 3: 259-263.

9. Abdulaal OM, Rainford L, MacMahon P, Kavanagh E, Galligan M, Cashman J. 3T MRI of the knee with optimized isotropic 3D sequences: accurate delineation of intra-articular pathology without prolonged acquisition times. Eur Soc Radiol 2017; 1-8.

10. Kataoka M, Ueda H, Koyama T, Umeoka S, Togashi K, Asato R. Contrast enhanced volumetric interpolated breath-hold examination compared with spin-echo T1- weighted imaging of head and neck tumors. Am J Roentgenol 2005; 184: 313-319.

11. Crema MD, Roemer FW, Marra MD, Burstein D, Gold GE, Eckstein F. Articular cartilage in the knee: current MR imaging techniques and applications in clinical practice and research. Radiograph 2011; 31: 37-61.

12. Govind BC, Paul SB, Bhavin GJ, Hai-Ling MC, Manohar MS. Steady-state MR imaging sequences: physics, classification, and clinical applications. Radiograph 2008; 28: $1147-1160$.

13. Arce K. Imaging findings in bisphosphonate- related osteonecrosis of jaws. J Oral Maxillofac Surg Off J Am Assoc Oral Maxillofac Surg 2009; 67: 75-84.

14. Lazik-Palm A, Kraff O, Geis C, Johst S, Goebel J, Ladd ME. Morphological imaging and T2 and T2* mapping of hip cartilage at 7 Tesla MRI under the influence of intravenous gadolinium. Eur Soc Radiol 2016; 26: 3923-3931.

15. Notohamiprodjo M, Horng A, Kuschel B, Paul D, Li G, Raya JG, Reiser MF, Glaser C. 3D-imaging of the knee with an optimized 3D-FSE-sequence and a 15-channel knee-coil. Eur J Radiol 2012; 81: 3441-3449.

16. Moriya S, Miki Y, Matsuno Y, Okada M. Threedimensional double-echo steady-state (3D-DESS) magnetic resonance imaging of the knee: establishment of flip angles for evaluation of cartilage at $1.5 \mathrm{~T}$ and $3.0 \mathrm{~T}$. Acta Radiologica 2012; 53: 790-794.

17. Moriya S, Miki Y, Yokobayashi T, Ishikawa M. Threedimensional double-echo steady-state (3D-DESS) magnetic resonance imaging of the knee: contrast optimization by adjusting flip angle. Acta Radiol 2009; 50: 507-511.

18. Notohamiprodjo M, Kuschel B, Horng A, Paul D, Baer P, Li G, Garcia del Olmo JM, Reiser MF, Glaser C. 3D-MRI of the ankle with optimized 3D-SPACE. Invest Radiol 2012; 47: 231-239.

19. McCauley TR, Disler DG. Magnetic resonance imaging of articular cartilage of the knee. J Am Acad Orthop Surg 2001; 9: 2-8.

20. Cicuttini F, Forbes A, Asbeutah A, Morris K, Stuckey S. Comparison and reproducibility of fast and conventional spoiled gradient echo magnetic resonance sequences in the determination of knee cartilage volume. J Orthop Res 2000; 18: 580-584.

21. Lee SY, Jee WH, Kim SK, Koh IJ, Kim JM. Differentiation between grade 3 and grade 4 articular cartilage defects of the knee: fat-suppressed proton density-weighted versus fat-suppressed three-dimensional gradient-echo MRI. Acta Radiol 2010; 51: 455-461.

22. Sylvain RD, Christian WAP, Marius RS, Marco Z, Peter PK, Fabian K. Articular Cartilage defects detected with 3D water-excitation true FISP: prospective comparison with sequences commonly used for knee imaging. Radiol 2007; 245.

23. Sylvain RD, Peter K, Marius RS, Wilhelm H, Juerg H, Christian WAP. Diagnosis of articular cartilage 
abnormalities of the knee: prospective clinical evaluation of a 3D water-excitation true FISP sequence. Radiol 2007; 243.

24. Notohamiprodjo M, Kuschel B, Horng A, Paul D, Baer P, Li G, Garcia del Olmo JM, Reiser MF, Glaser C. 3D-MRI of the ankle with optimized 3D-SPACE. Invest Radiol 2012; 47: 231-239.

25. Friedrich KM, Reiter G, Kaiser B, Mayerhofer M, Deimling M, Jellus V. High-resolution cartilage imaging of the knee at 3T: Basic evaluation of modern isotropic 3D MR-sequences. Eur J Radiol 2010; 78: 398-405.

26. Ali P, Mehdi R, Noradin G. A hybrid neural network-gray wolf optimization algorithm for melanoma detection. Biomed Res 2017; 28: 3408-3411.

27. Eckstein F, Reiser M, Englmeier KH, Putz R. In vivo morphometry and functional analysis of human articular cartilage with quantitative magnetic resonance imaging from image to data, from data to theory. Anat Embryol (Berl) 2001; 203: 147-173.

28. Hudelmaier M, Glaser C, Hohe J, Englmeier KH, Reiser M. Age-related changes in the morphology and deformational behavior of knee joint cartilage. Arthritis Rheum 2001; 44: 2556-2561.

29. Wirth W, Nevitt M, Hellio Le Graverand MP, Benichou O, Dreher D, Davies RY. Sensitivity to change of cartilage morphometry using coronal FLASH, sagittal DESS, and coronal MPR DESS protocols-comparative data from the
Osteoarthritis Initiative (OAI). Osteoarthr Cartil 2010; 18: 547-554.

30. Eckstein F, Hudelmaier M, Wirth W, Kiefer B, Jackson R, $\mathrm{Yu}$ J. Double echo steady state magnetic resonance imaging of knee articular cartilage at 3 Tesla: a pilot study for the osteoarthritis initiative. Ann Rheum Dis 2006; 65: 433-441.

31. Weckbach S, Mendlik T, Horger W, Wagner S, Reiser MF. Quantitative assessment of patellar cartilage volume and thickness at 3.0 Tesla comparing a 3D-fast low angle shot versus a 3D-true fast imaging with steady-state precession sequence for reproducibility. Invest Radiol 2006; 41: 189197.

32. Gold GE, McCauley TR, Gray ML, Disler DG. Whats new in cartilage? Radiographics 2003; 23: 1227-1242.

\section{*Correspondence to}

Mokhtar Mars

Université Tunis EL Manar

Institut Supérieur des Technologies Médicales de Tunis

Laboratoire de Recherche de Biophysique et de Technologies Médicales

Tunisia 